Alfarama Journal of Basic \& Applied Sciences

Faculty of Science Port Said University https://ajbas.journals.ekb.eg

ajbas@sci.psu.edu.eg

http://sci.psu.edu.eg/en/

January 2021, Volume 2, Issue 1

DOI: $10.21608 /$ ajbas.2020.43968.1035

Submitted: 27-09-2020

Accepted: 25-10-2020

Pages: $149-167$

\title{
Evaluation of Pesticide Residues in some Commonly Consumed Vegetables in Egypt and their Related Chronic Exposure
}

\author{
Ahmed Ismail Abdelkader ${ }^{1 * *}$, Mohsen Mohamed Ayoub ${ }^{2}$, Ali Saad Mohammed ${ }^{3}$ \\ ${ }^{1}$ Prof. of Environmental Microbiology,Department of Environmental Science, Faculty of Science, \\ Port Said University, 42522, Egypt \\ ${ }^{2}$ Researcher Professor, Central Lab. of Residue Analysis of Pesticides and Heavy Metals in Foods \\ (QCAP), Agricultural Research Center, Ministry of Agriculture and Land Reclamation, 7 Nadi \\ El- Said Street, Dokki, Giza, 12311, Egypt \\ ${ }^{3} \mathrm{Head}$ of pesticide residues section, Central Food Laboratories, Ministry of Public Health, Doha, 42, \\ Qatar
}

Corresponding author: ali_chemist84@hotmail.com

\begin{abstract}
A multi-residue quantitative method in combination with liquid and gas chromatography - tandem mass spectrometry, was employed to monitor 281 pesticide residus in the most consumed vegetables at the wholesale markets in Egypt during 2018. Among 506 samples analyzed, 66 different pesticide residues were detected in 405 samples (80\%) whereas, 260 samples $(51 \%)$ contaminated with levels lower than maximum residue limits and 145 samples (29\%) were violated. The highest violation was observed at $(60 \%, 58 \%, 46 \%$ and $33 \%)$ in parsley followed by pepper, beans and coriander, respectively. Chlorpyrifos, profenofos malathion, lambda-cyhalothrin and metalaxyl were the most frequently detected pesticides. Data Monitored was used for estimating the potential health risks associated with the exposures to the detected pesticides. The highest hazard index was found in tomato for emmactin benzoate $(10 \%)$ against the acceptable daily intake value for adults. Tomato was the most contributing commodity to the chronic exposure representing $56 \%$ to the total risk. The values of cumulative exposure for carbamates, heterocyclic, organophosphorus, pyrethroids, and other groups were calculated and found to be $<1$ (0.0060.256), indicating no risk to consumers due to the exposure to pesticide residues in Egyptian-fresh vegetables. The results showed that despite the high presence of pesticide residues in some vegetables, they did not pose any health risks to consumers.
\end{abstract}

Keywords:

Chronic Exposure, Cumulative Risk Assessment, Pesticide Residues, Vegetables.

\section{INTRODUCTION}

Fresh daily consumed vegetables are considered a follow-essential source for a healthy food and balanced diet [1]. Furthermore they rich in carbohydrates, vitamins, fibers, lipids and many of a supplementary nutrients. On the other hand agriculture crops are subject to attack by many diseases 
and pests that affect the yield and the quality of foodstuffs. Therefore, farmers worldwide use different groups of pesticides including organophosphorus, organochlrine, pyrethroids and carbamates in order to control diseases, protect crops, get high quality foodstuff to increase the agricultural production in line with population growth demands [2].

Pesticides are chemical compounds used during cultivation, post-harvest treatment, production and storage of agricultural products [3]. During their applications, pesticide residues have harmful, longterm and lethal effects on human health like heart diseases and Alzheimer's [4\&5]. Also, contaminants resulting from pesticide residues have become one of the most critical hazards on humanity due to their chronic and acute impact as headaches, nausea, cancer, and endocrine disruption [6]. In 1990, the WHO announced 220 case death of three million cases representing the exposure values of chronic and acute poisoning in the world. However, misuse and application of pesticides are widely used in both the developed and developing countries [7].

In Egypt, different classes of pesticides (fungicides, insecticides, and herbicides) are used during the integrated pest management and/or pest control. However, many of farmers are spraying different pesticides against pests but ignoring pre-harvest intervals (PHI) due to their adequate un-awareness about the use of pesticides in pest control for various vegetable families [8]. Suc consume vegtable families eg. Cucurbitacea (cucumber), Solanaceae (tomato, pepper and eggplant) and leafy vegetables (lettuce, corinader and parsley) are daily consumed by many Egyptian. Leafy vegetables were reported to have violated pesticides exceeding the maximum residue limits (MRL) [9]. The most frequently detected pesticide in vegetables collected from Egyptian markets were the OP's and PY's residues [10].

In the developing countries, the main tools for the monitoring survey of pesticide residues in fruits and vegetables, from equipment, control programs and training of technical personnel are often lacking [11].Therefore, the monitoring of human exposure to pesticide residues is needed to conduct through comparing of residue levels with the international standards such as maximum residue limits (MRLs) that set by the Codex Alimentarius, European (EU) commissions and to acceptable daily intakes (ADIs) set by FAO/WHO. The acceptable daily intake is the permitted amount of chemicals in food expressed as ( $\mathrm{mg} / \mathrm{kg}$ body weight per day) that the human body can be exposed to it daily over a period of time life [12].The monitoring research are always interested in good agriculture practice of pesticides (GAP) such as the registration, authorization, application and compliance with MRL's [13]. Therfore, standard methods to assess the hazards due to pesticide residues and to evaluate the chronic and acute exposure are basde on the average estimated daily intakes per day [14].

The aim of the present study was to assess the concentration levels of residues detected in the most popular consumed vegetables of Egyptian wholesale markets during 2018. To evaluate the compliance of obtained results with MRL's set by Codex and/or EU [15-16]. The obtained monitoring results also evaluated to check the application of Good Agriculture Practice (GAP) and to estimate the dietary exposure and compared with safety limits such as the ADI and/or (ARfD) set by both FAO and WHO.

\section{MATERIALS AND METHODS}

\subsection{Sampling}

A total of 506 samples from 10 different vegetables of the most popular consumed and cultivated vegtables, four fruiting vegetables, i.e. cucumber, eggplant, peper and tomato; three leafy vegetables, i.e. coriander, parsley and lettuce; one root vegetable (carrot), one bulb onion and one legume bean were collected from two major wholesale markets (Obour \& Six October) in Egypt during 2018. One to two kilograms of each plant crop were prepared for examination against 281 pesticide residues according to Codex Alimentarius Commission (CAC) guidelines, 1993 [17]. The samples were kept 
in polythene bags and then transported on ice to the laboratory where they were analyzed immediately and / or grinded with high speed grinder 2 litter capacities jar with lid and stored at $0-5{ }^{0} \mathrm{C}$ for no longer than 2 days before analysis.

\subsection{Selection of Targeted Pesticides and Analytical Multi-Residue Method}

A total of 281 pesticides belong to four categories, insecticides (132), fungicides (76), herbicides (67) and acaricides (6) were monitored for detection of any trace levels of their residues in some commonly consumed market vegetables including beans, carrot, coriander, cucumber, eggplant, lettuce, onion, parsley, pepper and tomato. Pesticides were selected based on their either currently registered or banned and their commercial use in Egypt for controlling mites or ticks, fungi, unwanted weeds and insects with reference to Agricultural Pesticides Committee (APC).

A modified QuEChERS method EN 15662:2008, was used for extraction of samples and to minimize degradation of susceptible compounds (e.g. base and acid labile pesticides), expand the range of matrices amenable by this approach and increase sample throughput while reducing costs [18]. The procedures were carried out based on an initial single- phase extraction using a test homogeneous portion of $10 \mathrm{~g}$ sample (water content $>80 \%$ ) with acetonitrile at $10 \mathrm{ml} / 10 \mathrm{~g}$ of sample. A liquid -liquid partition is followed by adding one contents of QuEChERS extract kit, part no. 5982-5650. Centrifugation carried out at $10000 \mathrm{rpm}$ for 10 minutes. An aliquot of upper layer, containing pesticide was transferred to $15 \mathrm{ml}$ tube in which matrices were cleaned and the excess of water removed by mixing the acetonitrile (CAN) with magnesium sulfate anhydrous and PSA sorbents through Single d-SPE step, part no. 5982-5056. The final extract can be analyzed directly by either LC-MS/MS or GC-MS/MS technologies

\subsection{Instrumentations and Conditions}

\subsubsection{Reagents and Reference Standards}

All Pesticides active ingredients were obtained from Sigma Aldrich (Steinheim, Germany) with purity $\geq 95 \%$ and re-kept in refrigerator freezer at $-20{ }^{\circ} \mathrm{C}$. Stock standard solutions of $1000 \mu \mathrm{g} / \mathrm{mL}$ were prepared in $10 \mathrm{~mL}$ volumetric flask in acetonitrile and transferred to amber-screw-capped glass vial $(12 \mathrm{~mL})$. Pesticide Stock standard solutions were kept in the dark at $-20 \pm 2{ }^{\circ} \mathrm{C}$ [19]. Intermediate mixture standard solutions $(100,10$ and 1$) \mu \mathrm{g} / \mathrm{mL}$ was prepared in acetonitrile for LC-MS/MS and HA (9:1) in case of GC-MS/MS. A multi-level calibration mixture solution (5, 10, 25, 50 and 100) $\mu \mathrm{g} / \mathrm{L}$ were prepared from of $1000 \mu \mathrm{g} / \mathrm{L}$. Methanol, Acetonitrile (LC-MS grade), Hexane and Acetone (GC-MS grade). Ammonia solution 33\% was obtained from Honeywell (Riedel-de-Haën). Formic acid $98-100 \%$ (Merck) was used as acid modifier of the LC mobile phase. De-ionized water was generated by Milli-Q unit (Millipore Corporate, USA) to get LC-grade water. Buffer salts were purchased from Agilent technologies: QuEChERS extract kit, part no. 5982-5650, containing magnesium sulphate $4 \mathrm{~g}$; sodium chloride $1 \mathrm{~g}$; trisodium citrate $1 \mathrm{~g}$ and disodium hydrogen sesquihydrate $0.5 \mathrm{~g}$, with purity $\geq 99 \%$. Dispersive SPE $15 \mathrm{ml}$, part no. 5982-5056, was containing 25 $\mathrm{mg}$ of the primary secondary amine bulk sorbent (PSA) and $150 \mathrm{mg}$ magnesium sulphate with purity $\geq$ $98 \%$.

\subsubsection{LC-MS/MS Determination}

Acquity UPLC system equipped with binary pump, vacuum degasser, and auto sampler. gas generator designed to provide nitrogen gas at least $5 \mathrm{~L} / \mathrm{min}$ at $60 \mathrm{psi}$, source gas (zero air) at least 22 $\mathrm{L} / \mathrm{min}$ at $100 \mathrm{psi}$ and exhaust gas (air) at least $8 \mathrm{~L} / \mathrm{min}$ at $60 \mathrm{psi} . \mathrm{LC}-\mathrm{MS} / \mathrm{MS}$ was carried out using a Waters Xevo TQ-S (Waters systems) triple quadrupole mass spectrometer with mass range $\mathrm{m} / \mathrm{z} 50$ to 2000 and equipped with atmospheric pressure ion source (Turbo V). Separation was performed on ACQUITY BEF C18 $2.1 \times 100 \mathrm{~mm}, 1.7 \mu \mathrm{m}$ particle size. The injection volume was $2 \mu \mathrm{L}$. A gradient elution program at $0.45 \mathrm{ml} / \mathrm{min}$ flow, in which one reservoir contained $10 \mathrm{mM}$ ammonium format solution in methanol-water (1:9) and the other contained methanol. The ESI source was used in the 
positive mode, argon nebulizer and other gas setting were optimized according to recommendations made by the manufacturer; source temperature was $300^{\circ} \mathrm{C}$, ion spray potential, $5500 \mathrm{v}$, de-cluster potential and collision energy were optimizes using a Harvard apparatus syringe pump by introducing individual pesticide solutions into the MS instrument to allow optimization of the MS/MS conditions. The Multiple Reactions monitoring mode (MRM) was used in which one MRM was used for quantification and the other for confirmation.

\subsubsection{GC-MS/MS Determination}

Agilent gas chromatograph (7890A) equipped with triple quadrupole tandem mass spectrometer (7000B), EI source was introduced to perform analysis by using HP-5MS capillary column (30 m, 250 $\mu \mathrm{m}$ id, and $0.25 \mu \mathrm{m}$ film thickness). Samples were injected with the following conditions: constant pressure, $36.73 \mathrm{psi}$; inlet temperature, $280{ }^{\circ} \mathrm{C}$; injection volume, $3 \mu \mathrm{l}$ (splitless); initial oven temperature, $70{ }^{\circ} \mathrm{C}$, held for $2 \mathrm{~min}$, then a $25{ }^{\circ} \mathrm{C} / \mathrm{min}$ ramp to $150{ }^{\circ} \mathrm{C}$ and $3{ }^{\circ} \mathrm{C} / \mathrm{min}$ ramp to $200{ }^{\circ} \mathrm{C}$ followed by a $8{ }^{\circ} \mathrm{C} / \mathrm{min}$ ramp to $280^{\circ} \mathrm{C}$ and held for $10 \mathrm{~min}$.

\subsubsection{Method Performance and Quality Assurance}

To ensure the fitness of purpose for the method in accordance with European standard, the in-house method validation was carried out according to SANTE and Eurachem guideline's requirements and criteria. The method validation parameters including limit of detection (LOD), limit of quantification (LOQ), accuracy (precision and trueness), recovery method linearity and measurement uncertainty. LOQ's were $10 \mu \mathrm{g} / \mathrm{L}$ for all tested pesticides. The evaluation of linearity was performed in the range from $(5-100) \mu \mathrm{g} / \mathrm{L}$ and the method linearity for most of pesticides was with correlation coefficient (R2) $\geq 0.995$ and deviation of recalculations $\leq \pm 20 \%$. Mean recoveries for all target pesticides were in the range from $70-120 \%$ expressed as trueness/accuracy [20]. The reproducibility expressed as a relative standard deviation (RSD) was $\leq 20 \%$. The LOQ criteria should fulfil the requirements of precision and trueness/ accuracy regarding both recovery and RSD \%. Furthermore, LOQ values were almost $\leq$ the lowest MRL. Expanded uncertainty expressed as RSD \% and at confidence level 95\% was found to be $< \pm 50 \%$ (European default value) [21].

\subsection{Estimated Daily Intake (EDI) Calculations}

The exposure to the pesticide residues was evaluated via estimation of daily intake (EDI) and compared with toxicological criteria such as acceptable daily intakes (ADIs). EDI was calculated for each commodity/pesticide combinations using the following equation:

EDI $\left(\mathrm{mg} \mathrm{kg}^{-1} \mathrm{bw}\right.$ day $\left.\mathrm{y}^{-1}\right)=\left[\right.$ Mean Concentration $\left(\mathrm{mgkg}^{-1}\right)$ of pesticide residue X Amount of intake food $\left(\mathrm{kgd}^{-1}\right)$ per body weight $\left.(\mathrm{kg})\right]$ [22].

The evaluation of dietary exposure to pesticide residues was based on a total of detected residues and food consumption assumptions set by WHO/GEMS/Food Cluster diets, as showed in Table 1 . Egypt was introduced in cluster G06 as countries clustered based on statistical similarities between dietary patterns. [23]. Hazard index (HI) was calculated by comparing the EDI value to its corresponding of ADI, taking in consideration of $60 \mathrm{~kg}$ as average adult's weight body [24\&25]. The estimated daily intakes were used as an indication for health risks to consumers on long term. When $\mathrm{HI}>1$; there is a risk to the consumer and the food is unacceptable and vice versa [26]. Cumulative risk ( $\Sigma \mathrm{HI}$ 's) equals the summation of $\mathrm{HI}$ for detectable pesticide belonging to the same chemical group.

\subsection{Monitoring surveys}

\section{RESULTS AND DISCUSSION}

The number of samples analyzed per each vegetable crop, detectable pesticides with their mean concentration range in $\mathrm{mg} / \mathrm{kg}$ and violated compounds of each pesticide/commodity are illustrated in (Table 2). 
In the current study, the results of the monitoring were evaluated versus to rules of Egyptian Agriculture Pesticides Committee (APC) which are applied in Egypt stated that "pesticide residue levels should be compared with Codex Alimentarius as it's available and to the EU-MRL's in case of codex MRL's lack" and EPA in case of EU lack. These rules would maintain the safety of agricultural products either consumed locally or exported abroad.

Overall, 20\% (101 samples) of samples analyzed had no detectable residues. Whereas, $80 \%$ (405 samples) had detectable pesticide residues of which $29 \%$ contaminated at levels above the MRL's and $51 \%$ (260 samples) within limits.

The results of detectable residues (>LOQ) were included in calculation and therefore the results for values less than LOQ are considered zero.

\begin{tabular}{|c|l|c|c|}
\hline $\begin{array}{l}\text { Table } \\
\text { WHO/GEMS/food, Cluster G06 (Egypt) }\end{array}$ & Scientific \\
\hline S. & Vegetable & Scientific name & Consumption (g/day) \\
\hline 1 & Beans & Phaseolus vulgaris & 0.49 \\
\hline 2 & Carrot & Daucus carota & 6.1 \\
\hline 3 & Coriander & Coriandrum sativum & 0.17 \\
\hline 4 & Cucumber & Cucumis sativus & 34.92 \\
\hline 5 & Eggplant & Solanum melongena & 20.12 \\
\hline 6 & Lettuce & Lactuca sativa & 6.05 \\
\hline 7 & Onion & Allium cepa & 43.38 \\
\hline 8 & Parsley & Petroselinum crispum & 0.77 \\
\hline 9 & Pepper & Capsicum annuum & 6.24 \\
\hline 1 & Tomato & Solanum lycopersicum & 200.93 \\
\hline
\end{tabular}

The obtained results for the contamination and violation rates were summarized for each group of plant crops. Whereas, the highest contamination percentages were recorded for fruiting vegetables of $32 \%$, after that leafy vegetables $(25 \%)$, bulb vegetables $(11 \%)$ and then legume vegetables $(10 \%)$ of which $10 \%, 11 \%, 3 \%$ and $5 \%$ were violated i.e. exceed the permissible limits, respectively. No violation was observed for root vegetables i.e. carrot while 6\%, was observed for pepper and parsley, $5 \%$ for beans and $3 \%$ for coriander and onion (Table 3 ).

Table 2. The number of analyzed samples per each vegetable crop, frequency of residues levels, MRL's and their violated pesticides.

\begin{tabular}{|c|c|c|c|c|c|c|c|c|}
\hline \multirow[t]{2}{*}{ Pesticides residues detected } & \multirow[t]{2}{*}{ Freq } & \multirow[t]{2}{*}{$<\mathrm{LOQ}$} & Min & Max & Mean & $\begin{array}{l}\text { Codex } \\
\text { MRL }\end{array}$ & $\begin{array}{c}\text { EU } \\
\text { MRL }\end{array}$ & \multirow[t]{2}{*}{$\begin{array}{c}\text { Viol } \\
\text { Compounds }\end{array}$} \\
\hline & & & \multicolumn{5}{|c|}{$\mathrm{mg} / \mathrm{kg}$} & \\
\hline \multicolumn{9}{|c|}{ Commodity No. 1. Beans, Total analyzed sample: (60) } \\
\hline Acetamiprid & 8 & 1 & 0.010 & 0.080 & 0.036 & 0.40 & 0.30 & \\
\hline Azoxystrobin & 7 & 1 & 0.010 & 0.060 & 0.032 & 0.06 & 3.00 & \\
\hline Buprofezin & 1 & & 0.020 & 0.020 & 0.020 & 0.70 & 0.01 & 1 \\
\hline Carbendazim & 11 & 1 & 0.010 & 0.250 & 0.088 & & 0.20 & \\
\hline Chlorpyrifos & 13 & & 0.010 & 0.260 & 0.100 & & 0.01 & 9 \\
\hline Cypermethrin & 14 & & 0.010 & 0.290 & 0.080 & 0.07 & 0.70 & \\
\hline Cyprodinil & 5 & & 0.020 & 0.240 & 0.130 & 2.00 & 2.00 & \\
\hline Difenoconazole & 15 & & 0.010 & 0.140 & 0.056 & 0.70 & 1.00 & \\
\hline
\end{tabular}




\begin{tabular}{|c|c|c|c|c|c|c|c|c|}
\hline Dimethoate & 4 & & 0.020 & 0.210 & 0.078 & & 0.01 & 4 \\
\hline Dimethomorph & 2 & & 0.010 & 0.140 & 0.075 & 0.70 & 0.01 & 1 \\
\hline Diniconazole & 4 & & 0.010 & 0.060 & 0.033 & 0.01 & 0.01 & 3 \\
\hline Fenpropathrin & 2 & 1 & 0.020 & 0.020 & 0.020 & & 0.01 & 1 \\
\hline Fenpyroximate & 1 & & 0.030 & 0.030 & 0.030 & 0.20 & 0.70 & \\
\hline Flusilazole & 4 & & 0.010 & 0.030 & 0.020 & & 0.01 & 3 \\
\hline Hexythiazox & 1 & & 0.010 & 0.010 & 0.010 & & 0.50 & \\
\hline Imidacloprid & 8 & & 0.020 & 0.240 & 0.090 & 2.00 & 2.00 & \\
\hline Indoxacarb & 2 & & 0.030 & 0.030 & 0.030 & & 0.50 & \\
\hline Iprodione & 7 & & 0.010 & 1.000 & 0.298 & & 0.01 & 5 \\
\hline Lambda-Cyhalothrin & 9 & 1 & 0.010 & 0.020 & 0.015 & 0.30 & 0.40 & \\
\hline Malathion & 2 & & 0.030 & 0.090 & 0.060 & 1.00 & 0.02 & 2 \\
\hline Metalaxyl & 4 & 1 & 0.010 & 0.040 & 0.025 & & 0.20 & \\
\hline Myclobutanil & 3 & & 0.010 & 0.010 & 0.010 & 0.80 & 0.80 & \\
\hline Omethoate & 3 & & 0.010 & 0.020 & 0.015 & & 0.01 & 2 \\
\hline Penconazole & 1 & & 0.020 & 0.020 & 0.020 & & 0.01 & 1 \\
\hline Profenofos & 3 & & 0.010 & 0.020 & 0.015 & & 0.01 & 1 \\
\hline Propamocarb & 5 & & 0.010 & 0.080 & 0.037 & & 0.10 & \\
\hline Propiconazol & 1 & & 0.010 & 0.010 & 0.010 & & 0.01 & \\
\hline Pyridaben & 1 & & 0.010 & 0.010 & 0.010 & & 0.20 & \\
\hline Pyrimethanil & 1 & & 0.110 & 0.110 & 0.110 & & 3.00 & \\
\hline Spinosad & 2 & & 0.030 & 0.080 & 0.055 & & 0.30 & \\
\hline Tebuconazole & 1 & & 0.070 & 0.070 & 0.070 & 3.00 & 2.00 & \\
\hline Thiacloprid & 1 & & 0.580 & 0.580 & 0.580 & & 0.40 & \\
\hline Thiobencarb & 1 & & 0.010 & 0.010 & 0.010 & & 0.01 & \\
\hline Thiophanate-methyl & 5 & & 0.020 & 0.420 & 0.184 & & 0.10 & 2 \\
\hline Triadimenol & 1 & & 0.010 & 0.010 & 0.010 & 1.00 & 0.01 & \\
\hline
\end{tabular}

Commodity No. 2. Carrot, Total analyzed sample: (37)

\begin{tabular}{|c|c|c|c|c|c|c|c|c|}
\hline Chlorpyrifos & 3 & 1 & 0.010 & 0.160 & 0.085 & 0.10 & 0.10 & \\
\hline Difenoconazole & 1 & & 0.010 & 0.010 & 0.010 & 0.20 & 0.40 & \\
\hline Dimethomorph & 1 & & 0.010 & 0.010 & 0.010 & & 0.01 & \\
\hline Flutolanil & 1 & & 0.160 & 0.160 & 0.160 & & 0.01 & 1 \\
\hline Malathion & 1 & & 0.010 & 0.010 & 0.010 & & 0.02 & \\
\hline Myclobutanil & 1 & & 0.010 & 0.010 & 0.010 & & 0.20 & \\
\hline Omethoate & 1 & & 0.010 & 0.010 & 0.010 & & 0.02 & \\
\hline Penconazole & 1 & & 0.010 & 0.010 & 0.010 & & 0.01 & \\
\hline Propamocarb & 1 & & 0.010 & 0.010 & 0.010 & & 0.01 & \\
\hline Pyridaben & 1 & & 0.010 & 0.010 & 0.010 & & 0.01 & \\
\hline
\end{tabular}

\begin{tabular}{|l|c|c|c|c|c|c|c|}
\hline Commodity No. 3. Coriander, Total analyzed sample: $(\mathbf{6 0})$ & & & & \\
\hline Acetamiprid & 3 & & 0.010 & 0.010 & 0.010 & 0.05 & \\
\hline Atrazine & 2 & & 0.010 & 0.560 & 0.285 & 0.10 & 3 \\
\hline Carbendazim & 16 & 1 & 0.010 & 0.350 & 0.133 & 0.10 & \\
\hline Chlorpyrifos & 39 & 9 & 0.010 & 1.700 & 0.376 & 5.00 & \\
\hline Cypermethrin & 3 & 1 & 0.010 & 0.020 & 0.015 & 0.10 & \\
\hline Diazinon & 2 & & 0.010 & 0.010 & 0.010 & 5.00 & \\
\hline Difenoconazole & 1 & & 0.040 & 0.040 & 0.040 & 0.30 & \\
\hline
\end{tabular}




\begin{tabular}{|c|c|c|c|c|c|c|c|c|}
\hline Fenarimol & 1 & & 0.010 & 0.010 & 0.010 & & 0.05 & \\
\hline Flusilazole & 5 & 2 & 0.010 & 0.010 & 0.010 & & 0.05 & \\
\hline Hexaconazole & 1 & & 0.020 & 0.020 & 0.020 & & 0.05 & \\
\hline Imidacloprid & 1 & & 0.070 & 0.070 & 0.070 & & 0.05 & \\
\hline Iprodione & 1 & & 0.050 & 0.050 & 0.050 & & 0.05 & \\
\hline Lambda-Cyhalothrin & 4 & & 0.010 & 0.050 & 0.030 & & 0.01 & 1 \\
\hline Malathion & 22 & 4 & 0.010 & 0.380 & 0.108 & & 0.02 & 6 \\
\hline Metalaxyl & 12 & 3 & 0.010 & 0.080 & 0.036 & & 0.05 & \\
\hline Myclobutanil & 2 & & 0.010 & 0.010 & 0.010 & & 0.05 & \\
\hline Oxyfluorfen & 1 & & 0.010 & 0.010 & 0.010 & & 0.05 & \\
\hline Penconazole & 9 & & 0.010 & 0.110 & 0.050 & & 0.05 & \\
\hline Pendimethalin & 20 & 3 & 0.010 & 0.550 & 0.185 & & 0.05 & 8 \\
\hline Profenofos & 27 & 4 & 0.010 & 1.000 & 0.238 & & 0.05 & 9 \\
\hline Propamocarb & 1 & & 0.050 & 0.050 & 0.050 & & 0.05 & \\
\hline Propiconazol & 2 & & 0.140 & 0.560 & 0.350 & & 0.05 & 2 \\
\hline Tebuconazole & 3 & & 0.010 & 0.030 & 0.020 & & 1.50 & \\
\hline Thiophanate-methyl & 1 & & 0.080 & 0.080 & 0.080 & & 0.10 & \\
\hline Triadimenol & 1 & & 0.010 & 0.010 & 0.010 & & 0.05 & \\
\hline \multicolumn{9}{|c|}{ Commodity No. 4. Cucumber, Total analyzed sample: (43) } \\
\hline Acetamiprid & 2 & & 0.020 & 0.090 & 0.055 & 0.30 & 0.30 & \\
\hline Azoxystrobin & 3 & & 0.050 & 1.800 & 0.925 & 1.00 & 1.00 & 1 \\
\hline Benalaxyl & 1 & & 0.020 & 0.020 & 0.020 & & 0.05 & \\
\hline Carbendazim & 1 & & 0.020 & 0.020 & 0.020 & 0.50 & 0.10 & \\
\hline Carbofuran & 1 & & 0.030 & 0.030 & 0.030 & & 0.02 & 1 \\
\hline Chlorpropham & 1 & & 0.010 & 0.010 & 0.010 & & 0.01 & \\
\hline Chlorpyrifos & 2 & & 0.020 & 0.060 & 0.040 & & 0.01 & 2 \\
\hline Cypermethrin & 1 & & 0.100 & 0.100 & 0.100 & 0.07 & 0.20 & \\
\hline Dimethomorph & 2 & & 0.010 & 0.070 & 0.040 & 0.50 & 0.50 & \\
\hline Ethion & 1 & & 0.060 & 0.060 & 0.060 & & 0.01 & 1 \\
\hline Flutolanil & 1 & & 0.010 & 0.010 & 0.010 & & 0.01 & \\
\hline Hexythiazox & 2 & & 0.020 & 0.240 & 0.130 & 0.05 & 0.50 & 1 \\
\hline Imidacloprid & 1 & & 0.020 & 0.020 & 0.020 & 1.00 & 1.00 & \\
\hline Indoxacarb & 1 & & 0.010 & 0.010 & 0.010 & 0.50 & 0.50 & \\
\hline Iprodione & 1 & & 0.190 & 0.190 & 0.190 & 2.00 & 0.01 & 1 \\
\hline Malathion & 1 & & 0.010 & 0.010 & 0.010 & 0.20 & 0.02 & \\
\hline Mandipropamid & 2 & & 0.020 & 0.040 & 0.030 & 0.20 & 0.20 & \\
\hline Metalaxyl & 7 & 1 & 0.010 & 0.260 & 0.082 & 0.50 & 0.50 & \\
\hline Myclobutanil & 1 & & 0.020 & 0.020 & 0.020 & 0.20 & 0.20 & \\
\hline Profenofos & 1 & & 1.060 & 1.060 & 1.060 & & 0.01 & 1 \\
\hline Propamocarb & 9 & & 0.030 & 0.530 & 0.213 & 5.00 & 5.00 & \\
\hline Propiconazol & 2 & & 0.010 & 0.020 & 0.015 & & 0.01 & 1 \\
\hline Tetraconazole & 1 & & 0.010 & 0.010 & 0.010 & & 0.20 & \\
\hline Thiamethoxam & 3 & & 0.010 & 0.070 & 0.033 & 0.50 & 0.50 & \\
\hline Thiophanate-methyl & 1 & & 0.120 & 0.120 & 0.120 & & 0.10 & \\
\hline Trifloxystrobin & 1 & & 0.040 & 0.040 & 0.040 & & 0.30 & \\
\hline
\end{tabular}




\begin{tabular}{|l|l|l|l|l|l|l|l|l|}
\hline Commodity No. 5. Eggplant, Total analyzed sample: & $(\mathbf{4 4})$ \\
\hline Acetamiprid & 5 & & 0.010 & 0.380 & 0.176 & 0.20 & 0.20 & 1 \\
\hline Azoxystrobin & 3 & 2 & 0.020 & 0.020 & 0.020 & 3.00 & 3.00 & \\
\hline Carbendazim & 1 & & 0.010 & 0.010 & 0.010 & & 0.50 & \\
\hline Chlorpyrifos & 3 & & 0.090 & 1.500 & 0.610 & & 0.01 & 3 \\
\hline Clothianidin & 1 & 1 & & $<$ LOQ & & & 0.04 & \\
\hline Diazinon & 1 & 1 & & $<$ LOQ & & & 0.01 & \\
\hline Dimethomorph & 1 & & 0.020 & 0.020 & 0.020 & 1.50 & 1.00 & \\
\hline Famoxadone & 2 & & 0.030 & 0.120 & 0.075 & & 1.50 & \\
\hline Fenpropathrin & 3 & & 0.070 & 0.340 & 0.247 & & 0.01 & 3 \\
\hline Imidacloprid & 2 & 1 & 0.750 & 0.750 & 0.750 & 0.20 & 0.50 & 1 \\
\hline Metalaxyl & 2 & 1 & 0.010 & 0.010 & 0.010 & & 0.01 & \\
\hline Myclobutanil & 1 & & 0.010 & 0.010 & 0.010 & & 0.30 & \\
\hline Profenofos & 1 & & 0.010 & 0.010 & 0.010 & & 0.01 & \\
\hline Thiamethoxam & 5 & 1 & 0.010 & 0.050 & 0.030 & 0.70 & 0.20 & \\
\hline
\end{tabular}

\begin{tabular}{|c|c|c|c|c|c|c|c|c|}
\hline \multicolumn{9}{|c|}{ Commodity No. 6. Lettuce, Total analyzed sample: (22) } \\
\hline Atrazine & 2 & & 0.140 & 0.240 & 0.190 & & 0.05 & 2 \\
\hline Carbendazim & 3 & & 0.260 & 1.200 & 0.700 & 5.00 & 0.10 & 2 \\
\hline Chlorpyrifos & 3 & 1 & 0.010 & 0.010 & 0.010 & & 0.01 & \\
\hline Imidacloprid & 7 & 1 & 0.020 & 0.080 & 0.054 & 2.00 & 2.00 & \\
\hline Iprodione & 3 & 1 & 0.010 & 0.020 & 0.015 & 10.00 & 0.01 & \\
\hline Lambda-Cyhalothrin & 1 & & 0.050 & 0.050 & 0.050 & & 0.15 & \\
\hline Malathion & 1 & 1 & & $<\mathrm{LOQ}$ & & & 0.50 & \\
\hline Metalaxyl & 3 & & 0.020 & 0.040 & 0.030 & 2.00 & 3.00 & \\
\hline Omethoate & 1 & & 0.010 & 0.010 & 0.010 & & 0.01 & \\
\hline Propamocarb & 1 & & 0.020 & 0.020 & 0.020 & 100.00 & 40.00 & \\
\hline
\end{tabular}

\begin{tabular}{|c|c|c|c|c|c|c|c|c|}
\hline \multicolumn{9}{|c|}{ Commodity No. 7. Onion, Total analyzed sample: (60) } \\
\hline Acetamiprid & 1 & & 0.100 & 0.100 & 0.100 & 0.02 & 0.01 & 1 \\
\hline Azoxystrobin & 15 & 1 & 0.020 & 1.510 & 0.261 & & 10.00 & \\
\hline Carbendazim & 5 & 1 & 0.010 & 0.040 & 0.025 & & 0.10 & \\
\hline Chlorpyrifos & 9 & 1 & 0.010 & 0.020 & 0.015 & 0.20 & 0.20 & \\
\hline Cypermethrin & 4 & & 0.090 & 0.130 & 0.110 & & 0.05 & 4 \\
\hline Cyprodinil & 2 & 2 & & $<\mathrm{LOQ}$ & & 0.30 & 0.30 & \\
\hline Diazinon & 2 & & 0.110 & 0.170 & 0.140 & 0.05 & 0.05 & 2 \\
\hline Difenoconazole & 1 & & 0.170 & 0.170 & 0.170 & 0.10 & 0.50 & 1 \\
\hline Dimethomorph & 12 & 2 & 0.010 & 0.130 & 0.046 & 0.60 & 0.60 & \\
\hline Emamectin benzoate & 1 & & 0.010 & 0.010 & 0.010 & & 0.01 & \\
\hline Flutolanil & 2 & & 0.020 & 0.030 & 0.025 & & 0.01 & 2 \\
\hline Imidacloprid & 2 & & 0.050 & 0.050 & 0.050 & 0.10 & 0.10 & \\
\hline Indoxacarb & 4 & & 0.020 & 0.080 & 0.043 & & 0.02 & \\
\hline Iprodione & 3 & & 0.030 & 0.160 & 0.093 & 0.20 & 0.01 & 3 \\
\hline Lambda-Cyhalothrin & 18 & & 0.010 & 0.250 & 0.090 & & 0.20 & \\
\hline Lufenuron & 1 & & 0.020 & 0.020 & 0.020 & & 0.01 & 1 \\
\hline Malathion & 3 & & 0.010 & 0.010 & 0.010 & 1.00 & 0.02 & \\
\hline Metalaxyl & 10 & 2 & 0.010 & 0.260 & 0.103 & 2.00 & 0.50 & \\
\hline Metribuzin & 1 & 1 & & $<\mathrm{LOQ}$ & & & 0.10 & \\
\hline
\end{tabular}




\begin{tabular}{|c|c|c|c|c|c|c|c|c|}
\hline Penconazole & 1 & 1 & & $<$ LOQ & & & 0.01 & \\
\hline Pendimethalin & 6 & 2 & 0.010 & 0.020 & 0.015 & 0.05 & 0.05 & \\
\hline Profenofos & 7 & & 0.010 & 1.300 & 0.484 & & 0.02 & 4 \\
\hline Propamocarb & 7 & & 0.010 & 0.540 & 0.144 & 2.00 & 2.00 & \\
\hline Spinosad & 1 & & 0.150 & 0.150 & 0.150 & 0.10 & 0.07 & \\
\hline Thiacloprid & 2 & & 0.010 & 0.020 & 0.015 & & 0.01 & 1 \\
\hline Thiophanate-methyl & 1 & & 0.040 & 0.040 & 0.040 & & 0.10 & \\
\hline Trifloxystrobin & 1 & & 0.020 & 0.020 & 0.020 & & 0.01 & 1 \\
\hline \multicolumn{9}{|c|}{ Commodity No. 8. Parsley, Total analyzed sample: (60) } \\
\hline Acetamiprid & 3 & & 0.020 & 0.190 & 0.083 & & 3.00 & \\
\hline Carbendazim & 4 & 1 & 0.010 & 0.020 & 0.015 & & 0.10 & \\
\hline Chlorpyrifos & 33 & 4 & 0.020 & 1.000 & 0.206 & & 0.02 & 28 \\
\hline Cypermethrin & 3 & & 0.010 & 0.010 & 0.010 & 0.70 & 2.00 & \\
\hline Diazinon & 1 & 1 & & $<\mathrm{LOQ}$ & & & 0.02 & \\
\hline Dimethoate & 1 & & 0.320 & 0.320 & 0.320 & & 0.02 & 1 \\
\hline Ethion & 1 & 1 & & $<\mathrm{LOQ}$ & & & 0.01 & \\
\hline Ethoprophos & 1 & & 0.010 & 0.010 & 0.010 & & 0.02 & \\
\hline Flusilazole & 1 & & 0.010 & 0.010 & 0.010 & & 0.02 & \\
\hline Lambda-Cyhalothrin & 13 & 1 & 0.010 & 0.430 & 0.135 & & 0.70 & \\
\hline Malathion & 38 & 7 & 0.010 & 3.650 & 0.647 & & 0.02 & 15 \\
\hline Metalaxyl & 5 & 3 & 0.020 & 0.030 & 0.025 & & 3.00 & \\
\hline Penconazole & 2 & 1 & 0.020 & 0.020 & 0.020 & & 0.02 & \\
\hline Pendimethalin & 8 & & 0.030 & 0.580 & 0.173 & & & \\
\hline Profenofos & 36 & 5 & 0.010 & 2.550 & 0.396 & & & 4 \\
\hline Propiconazol & 1 & & 0.010 & 0.010 & 0.010 & & 0.02 & \\
\hline Thiophanate-methyl & 5 & & 0.010 & 0.100 & 0.054 & & 0.10 & \\
\hline \multicolumn{9}{|c|}{ Commodity No. 9. Pepper, Total analyzed sample: (60) } \\
\hline Acetamiprid & 22 & 1 & 0.010 & 0.490 & 0.155 & 0.20 & 0.30 & 2 \\
\hline Azoxystrobin & 3 & & 0.020 & 0.110 & 0.053 & 3.00 & 3.00 & \\
\hline Bifenthrin & 2 & & 0.010 & 0.040 & 0.025 & & 0.50 & \\
\hline Buprofezin & 3 & 1 & 0.030 & 0.540 & 0.285 & & 0.01 & 2 \\
\hline Carbendazim & 3 & & 0.010 & 0.050 & 0.030 & & 0.10 & \\
\hline Carbofuran & 1 & & 0.010 & 0.010 & 0.010 & & 0.002 & 1 \\
\hline Carbosulfan & 1 & 1 & & $<$ LOQ & & & 0.002 & \\
\hline Chlorpropham & 1 & & 0.030 & 0.030 & 0.030 & & 0.01 & 1 \\
\hline Chlorpyrifos & 32 & 8 & 0.010 & 7.400 & 0.705 & 2.00 & 0.01 & 19 \\
\hline Cypermethrin & 16 & 4 & 0.010 & 0.200 & 0.080 & 0.10 & 0.50 & 1 \\
\hline Difenoconazole & 3 & & 0.040 & 0.280 & 0.137 & 0.60 & 0.90 & \\
\hline Dimethoate & 2 & 1 & 0.010 & 0.010 & 0.010 & 0.50 & 0.01 & \\
\hline Diniconazole & 1 & & 0.050 & 0.050 & 0.050 & & 0.01 & 1 \\
\hline Ethion & 1 & & 0.450 & 0.450 & 0.450 & & 0.01 & 1 \\
\hline Fenpropathrin & 2 & & 0.010 & 0.030 & 0.020 & & 0.01 & 1 \\
\hline Hexythiazox & 2 & 1 & 0.020 & 0.020 & 0.020 & & 0.50 & \\
\hline Imidacloprid & 13 & 2 & 0.010 & 0.820 & 0.204 & & 1.00 & \\
\hline Indoxacarb & 2 & 1 & 0.020 & 0.020 & 0.020 & & 0.30 & \\
\hline Iprodione & 1 & & 0.270 & 0.270 & 0.270 & & 0.01 & 1 \\
\hline
\end{tabular}




\begin{tabular}{|c|c|c|c|c|c|c|c|c|}
\hline Lambda-Cyhalothrin & 25 & 3 & 0.010 & 0.210 & 0.072 & & & 2 \\
\hline Malathion & 4 & 1 & 0.030 & 0.030 & 0.030 & & 0.02 & 1 \\
\hline Metalaxyl & 1 & 1 & & $<\mathrm{LOQ}$ & & & 0.50 & \\
\hline Methamidophos & 2 & & 0.230 & 0.430 & 0.330 & & 0.01 & 2 \\
\hline Myclobutanil & 1 & & 0.030 & 0.030 & 0.030 & & 0.50 & \\
\hline Omethoate & 3 & 1 & 0.010 & 0.050 & 0.030 & & 0.01 & 1 \\
\hline Permethrin & 1 & & 0.030 & 0.030 & 0.030 & & 0.05 & \\
\hline Phosalone & 1 & & 0.370 & 0.370 & 0.370 & & 0.01 & 1 \\
\hline Profenofos & 13 & 3 & 0.010 & 0.780 & 0.223 & & 0.01 & 8 \\
\hline Propamocarb & 2 & 1 & 0.640 & 0.640 & 0.640 & 3.00 & 3.00 & \\
\hline Pyridaben & 7 & 4 & 0.010 & 0.020 & 0.015 & & 0.01 & 2 \\
\hline Pyrimethanil & 1 & & 0.890 & 0.890 & 0.890 & & 2.00 & \\
\hline Pyriproxyfen & 1 & & 0.020 & 0.020 & 0.020 & & 1.00 & \\
\hline Spirodiclofen & 1 & & 0.080 & 0.080 & 0.080 & 0.20 & 0.20 & \\
\hline Tebuconazole & 1 & & 0.350 & 0.350 & 0.350 & 1.00 & 0.60 & \\
\hline Thiacloprid & 3 & & 0.010 & 0.020 & 0.015 & 1.00 & 1.00 & \\
\hline Thiamethoxam & 5 & & 0.030 & 0.240 & 0.125 & 0.70 & 0.70 & \\
\hline Thiophanate-methyl & 8 & & 0.040 & 0.860 & 0.346 & & 0.10 & 5 \\
\hline Triadimenol & 1 & & 0.290 & 0.290 & 0.290 & 1.00 & 0.50 & \\
\hline Trifloxystrobin & 2 & & 0.100 & 0.180 & 0.140 & 0.30 & 0.40 & \\
\hline
\end{tabular}

Commodity No. 10. Tomato, Total analyzed sample: (60)

\begin{tabular}{|c|c|c|c|c|c|c|c|c|}
\hline Acetamiprid & 2 & & 0.010 & 0.030 & 0.020 & 0.20 & 0.50 & \\
\hline Azoxystrobin & 2 & 1 & 0.130 & 0.130 & 0.130 & 3.00 & 3.00 & \\
\hline Bromuconazole & 1 & & 0.020 & 0.020 & 0.020 & & 0.01 & 1 \\
\hline Carbendazim & 4 & 1 & 0.010 & 0.020 & 0.015 & 0.50 & 0.30 & \\
\hline Chlorpyrifos & 8 & & 0.010 & 0.230 & 0.100 & & 0.10 & 1 \\
\hline Cymoxanil & 1 & & 0.010 & 0.010 & 0.010 & & 0.40 & \\
\hline Cypermethrin & 4 & 1 & 0.010 & 0.050 & 0.033 & 0.20 & 0.50 & \\
\hline Cyprodinil & 1 & 1 & & $<\mathrm{LOQ}$ & & 2.00 & 1.50 & \\
\hline Diazinon & 7 & & 0.010 & 0.110 & 0.056 & 0.50 & 0.01 & 10 \\
\hline Difenoconazole & 2 & & 0.010 & 0.020 & 0.015 & 0.80 & 2.00 & \\
\hline Dimethoate & 1 & & 0.010 & 0.010 & 0.010 & & 0.01 & \\
\hline Dimethomorph & 5 & & 0.010 & 0.130 & 0.058 & 1.50 & 1.00 & \\
\hline Emamectin benzoate & 2 & & 0.010 & 0.020 & 0.015 & & 0.02 & \\
\hline Famoxadone & 3 & & 0.010 & 0.180 & 0.070 & 2.00 & 2.00 & \\
\hline Fluopicolide & 1 & 1 & & $<$ LOQ & & 1.00 & 1.00 & \\
\hline Imidacloprid & 3 & & 0.020 & 0.050 & 0.035 & 0.50 & 0.50 & \\
\hline Lambda-Cyhalothrin & 2 & & 0.010 & 0.010 & 0.010 & 0.30 & 0.70 & \\
\hline Metalaxyl & 5 & & 0.010 & 0.250 & 0.070 & 0.50 & 0.30 & \\
\hline Profenofos & 5 & & 0.030 & 0.270 & 0.093 & 10.00 & 10.00 & \\
\hline Propamocarb & 5 & & 0.060 & 26.400 & 7.420 & 2.00 & 4.00 & 2 \\
\hline Spinosad & 1 & 1 & & $<$ LOQ & & 0.30 & 0.70 & \\
\hline Tebuconazole & 1 & & 0.010 & 0.010 & 0.010 & 0.70 & 0.90 & \\
\hline Thiacloprid & 4 & & 0.010 & 0.040 & 0.023 & 0.50 & 0.50 & \\
\hline Thiamethoxam & 2 & 1 & 0.040 & 0.040 & 0.040 & 0.70 & 0.20 & \\
\hline Thiophanate-methyl & 3 & 1 & 0.050 & 0.130 & 0.090 & & 1.00 & \\
\hline
\end{tabular}




\begin{tabular}{|l|l|l|l|l|l|l|}
\hline Trifloxystrobin & 1 & 0.090 & 0.090 & 0.090 & 0.70 & 0.70 \\
\hline
\end{tabular}

\begin{tabular}{|c|c|c|c|c|c|}
\hline Crops Groups & Commodities & Total & Free (ND) & Contaminated not violated & Violated \\
\hline \multirow{4}{*}{ Fruiting vegetables } & Cucumber & 43 & 14 & 22 & 7 \\
\hline & Eggplant & 44 & 16 & 23 & 5 \\
\hline & Pepper & 60 & 5 & 23 & 32 \\
\hline & Tomato & 60 & 8 & 44 & 8 \\
\hline \multicolumn{2}{|c|}{ Total } & 207 & 43 & 112 & 52 \\
\hline \multicolumn{2}{|c|}{$\%$} & $41 \%$ & $8.5 \%$ & $22 \%$ & $10 \%$ \\
\hline \multirow[t]{3}{*}{ Leafy vegetables } & Coriander & 60 & 6 & 36 & 18 \\
\hline & Parsley & 60 & 5 & 22 & 33 \\
\hline & Lettuce & 22 & 7 & 12 & 3 \\
\hline \multicolumn{2}{|c|}{ Total } & 142 & 18 & 70 & 54 \\
\hline \multicolumn{2}{|c|}{$\%$} & $28 \%$ & $3.5 \%$ & $14 \%$ & $11 \%$ \\
\hline Root vegetables & Carrot & 37 & 27 & 9 & 1 \\
\hline \multicolumn{2}{|c|}{$\%$} & $7 \%$ & $5.5 \%$ & $2 \%$ & $0 \%$ \\
\hline Bulb vegetables & Onion & 60 & 5 & 41 & 14 \\
\hline \multicolumn{2}{|c|}{$\%$} & $12 \%$ & $1 \%$ & $8 \%$ & $3 \%$ \\
\hline Legume vegetables & Beans & 60 & 8 & 28 & 24 \\
\hline \multicolumn{2}{|c|}{$\%$} & $12 \%$ & $1.5 \%$ & $5 \%$ & $5 \%$ \\
\hline \multicolumn{2}{|c|}{ Total analyzed Vegetables } & 506 & 101 & 260 & 145 \\
\hline \multicolumn{2}{|c|}{$\%$} & $100 \%$ & $20 \%$ & $51 \%$ & $29 \%$ \\
\hline
\end{tabular}

\subsection{Evaluation of detected pesticide}

About 66 pesticide residues were detected in the analyzed vegetable samples. The highest detected pesticide residue was emmactin benzoate with contribution percentage of $18.26 \%$, while bromuconazole recoded the lowest contribution percentage of $1.06 \%$ (Figure 1). The contribution percentage of other detected pesticide residues ranged from $1.18 \%$ (imidacloprid) to $14.62 \%$ (chlorpyrifos) (Figure1). The contribution percentage was calculated by dividing of the hazard index of each pesticides/Sum of total hazard indices. 


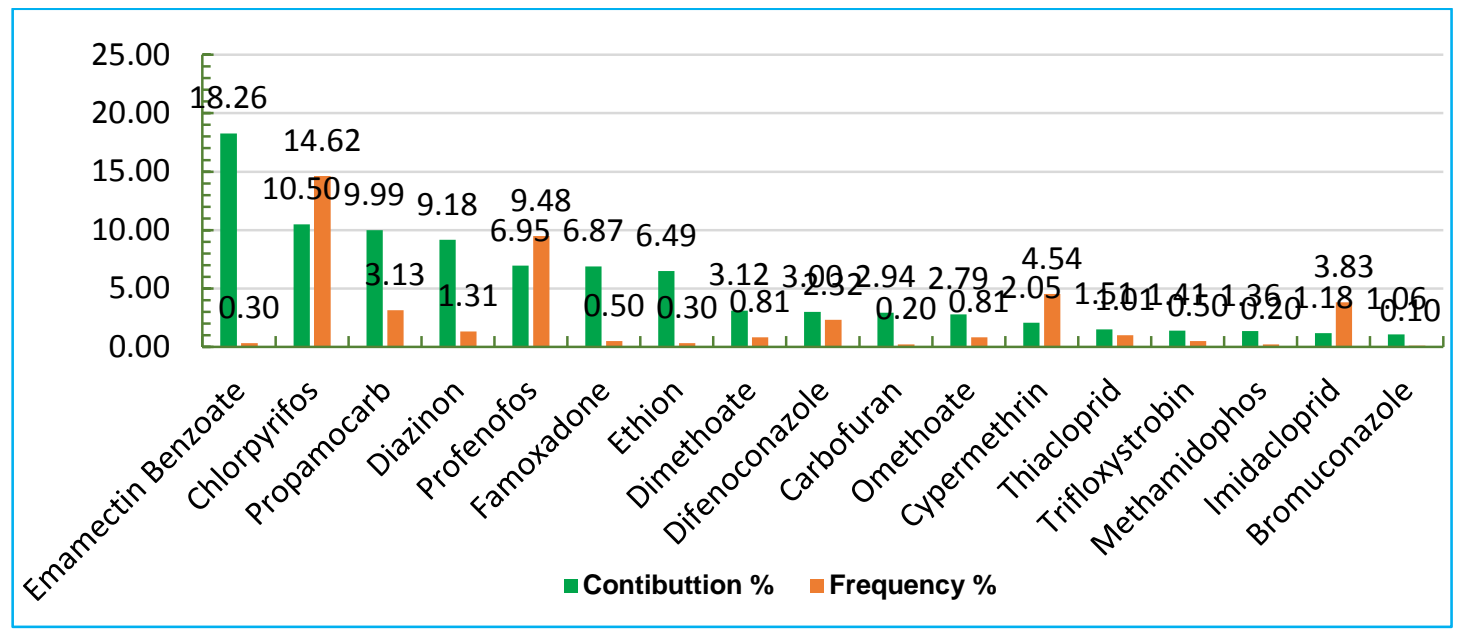

Figure1. The contribution $\%$ of pesticides in total hazard indices of detected pesticides residues and their frequencies $\%,>1 \%$

Organophosphorous was the most detected group followed by heterocyclic, carbamates and pyrethoids. Chlorpyrifos is an organophosphorous insecticide with a broad-spectrum activity. However, its mode of action (Cholinesterase inhibition) can cause a potential toxicity in humans [27]. Chlorpyrifos was recorded as the highest detected residue with highest violation rates in 28 vegetable samples collected from Polish farmers' fields [28]. Moreover, both of chlorpyrifos and cypermethrin were commonly used as effective insecticides which affect the nervous system of insects through disrupting both axon and synaptic transmission of the nerve impulses [29].

In the current study, the detected pesticides are classified according to chemical groups and the mode of applications classes with their percentages of detections and were found as follow; four chemical groups of organophosphorous (15\%), organochlorine (9\%), pyrethoids (8\%), carbamates (11\%), heterocyclic (35\%) and (23\%) other groups (Figure 2). The major classes were insecticides (28 compounds) with percentage of $42 \%$, fungicides (27) $41 \%$, herbicides (9) $14 \%$ and acaricides (2) with percentage 3\%. These obtained results are in line with other previous published studies, whereas most detected pesticides collected from Riyadh, KSA were organophosphorous, organochlorine, pyrethoids and carbamates [30].

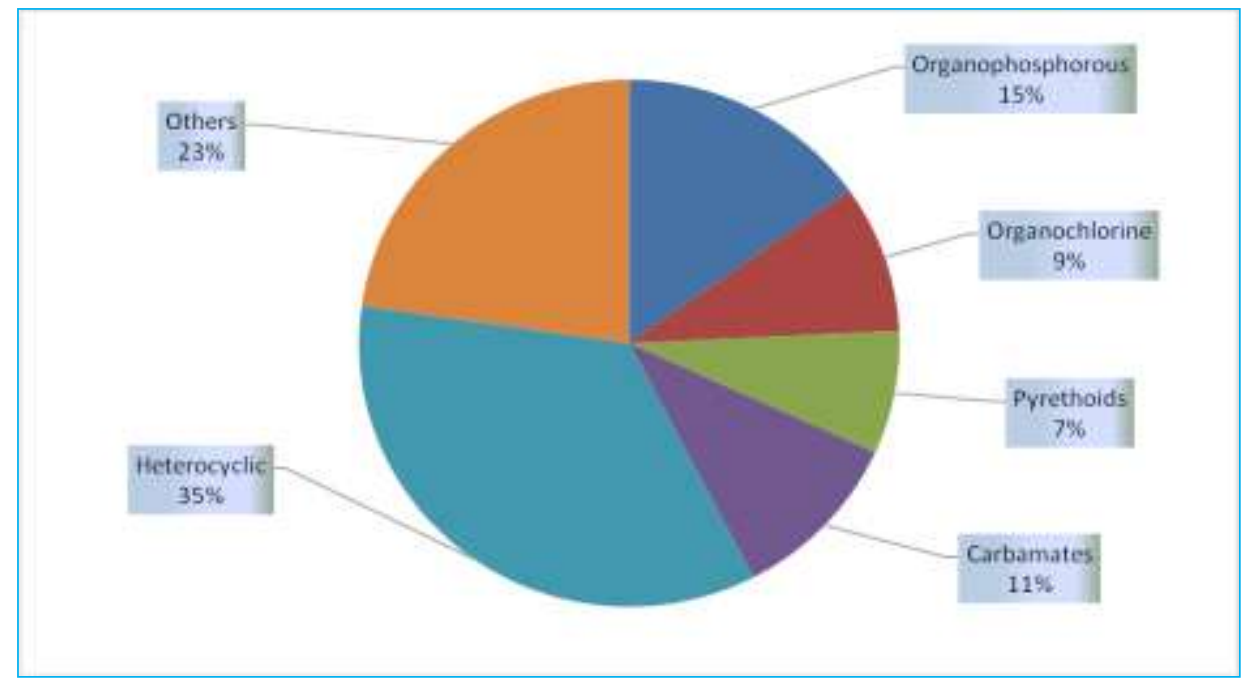

Figure 2. The contribution percentage of chemical groups of pesticide residues in the surveyed Egyptian vegetable crops 
The results also, showed that the highest contamination and violation rates were observed for leafy vegetables i.e. coriander, parsley, and lettuce. This might be due to the extensive and misuse of the applied pesticides especially on broad leaves.

In Egypt, leafy vegetables like molokhia and lettuce are cultivated in the field beside other crops which lead to high contamination due to the extensive use of pesticides [31 \& 32]. The current study is matched with compare to the presented study from Lucknow city, India, whereas, 23 pesticide residue with concentration range from $(0.005-12.35 \mathrm{mg} / \mathrm{kg})$. Moreover, some organochlorine pesticide residues which were banned in worldwide [33].

The monitoring survey of 180 samples collected from wholesale market in Korean, showed the presence of contamination rate of $6.7 \%$ was recorded for in 15 leafy vegetables [34].

\subsection{Chronic exposure assessment}

The long-term risk (chronic exposure) assessment requires a comparison between the exposure calculated with the mean pesticide residue levels consumed and the ADI.

Human health is subject to be at risk if the chronic exposure to toxic residues exceeded the acceptable daily intake on long term. However, in other reported there was health risk due to the residues of OP's i.e. chlorpyrifos, dicrovofos, monocrotophos and omethoate in some Ghanaian vegetables [35].

Dietary exposure was estimated for 66 pesticide residues with a detectable figure (not less than limit of quantification) to avoid overdose intake of contaminated food. The total exposure was evaluated by calculating all exposure cases of pesticide residues/food combinations. The estimated average daily intake (EDI ug $\mathrm{kg}^{-1} \mathrm{bw}^{-1}$ ) and the ratio of EDI to ADI for each pesticide residues i.e. hazard index (HI) were calculated. If the hazard index exceeds 1 , means the pesticide has exceeded the maximum acceptable level (e.g. ADI or ARfD) and there might thus be a risk. The obtains results illustrated in (Table 4).

Overall, the long-term exposure of the Egyptian consumers to pesticide residues through the consumption of vegetables appears to be relatively low. In most cases the HI's for detected pesticides were in the range of $(0.00028-11 \%)$. The highest $\mathrm{HI}$ was observed for emmactin benzoate $(18 \%$ of total HI's).

\subsection{Cumulative exposure}

Cumulative risk is one of the important approaches to evaluate hazard resulting from multiple residues. The methodology for cumulative risk assessment was used for the first time in 2010 by EFSA [36]. The presence of similar toxicological characteristics on food is the only case for the occurrence of cumulative effects.

Federal institute recommends evaluating the cumulative risk of pesticide residues via calculating the hazard indices for each pesticide belongs to the same chemical group. That is would provide us with adequate information about toxicity data to save the safety for the consumers [37]. The hazard index is a significant approach to evaluate the toxicological extent in food through comparing the ADL values with ADI and ARfD. Accordingly, determine the probability of any of risk through consumption of pesticide residues containing foodstuff.

In the current study, the values of Cumulative risk ( $\Sigma$ HR's) were obtained by calculating all HI for each pesticide belonging to the same chemical group.

The cumulative exposure values (hazard index) were found to be for, organophosphorous, pyrethoids, carbamates and heterocyclic <1 $(0.006-0.256)$ for adults, indicates no hazard to the consumers through the consumption of such vegetables (Table 4 ).

The obtained data showed that, the highest intakes were observed for organophosphates group followed by heterocyclic, carbamates and pyrethoids. However, the calculated intake indicated that, there was no risk associated to the consumption of vegetable samples whereas, all calculated HI for individual pesticides have values less than one indicated negligible risk. 
The contributions percentages were calculated to the total $\mathrm{HI}$, tomato was the most contributed commodity to HI with percentage of $56 \%$, followed by onion, cucumber, pepper and eggplant with contribution percentages of $13 \%, 12 \%, 11 \%$ and $5 \%$, respectively. Hence, there were relatively some levels of dietary exposure, lead to certain health risk to consumers. But reduced for leafy vegetables due to home washing and cooking process (Figure 3)

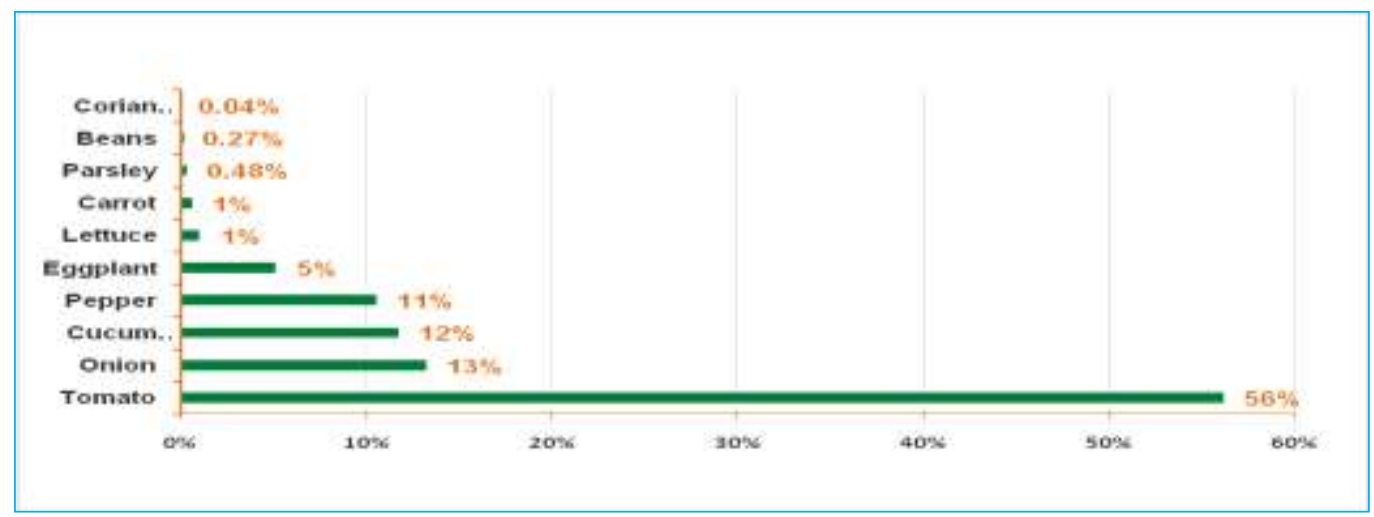

Figure 3. The $\%$ contribution of target commodity for the total hazard index $\%$

Organophosphate and pyrethroid pesticides were the major groups used in Egypt for more than 25 years (1980's), which refers to the exposure of consumers to these groups of pesticides for long periods that may be needed continuous evaluation of exposure via all sources such as food, environment, and water.

However, the exposure to lower levels of pesticides residues for a long periods were associated with harmful effects on human health such as cancer diseases and reproductive abnormalities [38].That is emphasized the need for new strategies to reduce dietary exposure to OP's PY's, carbamates, and other heterocyclic pesticides. Furthermore, introduce the bio pesticides in a farm work of integrated pest management (IPM)

\begin{tabular}{|c|c|c|c|c|c|c|c|}
\hline \multirow[t]{2}{*}{ SN } & Substance Group & Pesticides Detected & ADI & ARfD & Source & Total EDI & $\begin{array}{c}\text { HI } \\
\text { Total }\end{array}$ \\
\hline & & & $\begin{array}{c}\text { ug/kg.b } \\
\text { w }\end{array}$ & $\begin{array}{c}\text { ug/kg.b } \\
\text { w }\end{array}$ & & ug/ kg. bw & $\%$ \\
\hline \multirow[t]{10}{*}{1} & Organophosphorus & Chlorpyrifos & 10 & 100 & JMPR_2004 & 0.661 & 6.61053 \\
\hline & & Diazinon & 5 & 30 & JMPR_2006 & 0.289 & 5.77566 \\
\hline & & Dimethoate & 2 & 20 & JMPR_2003 & 0.039 & 1.96340 \\
\hline & & Ethion & 2 & 2000 & JMPR_1990 & 0.082 & 4.08600 \\
\hline & & Ethoprophos & 0 & 50 & JMPR_1999 & 0.000 & 0.03208 \\
\hline & & Malathion & 300 & 2000 & JMPR_2003 & 0.026 & 0.00876 \\
\hline & & Methamidophos & 4 & 10 & JMPR_2002 & 0.034 & 0.85800 \\
\hline & & Omethoate & 0 & 0 & JMPR_1985 & 0.005 & 1.75583 \\
\hline & & Phosalone & 20 & 300 & JMPR_2001 & 0.038 & 0.19240 \\
\hline & & Profenofos & 30 & 1000 & JMPR_2007 & 1.312 & 4.37274 \\
\hline \multicolumn{6}{|c|}{ 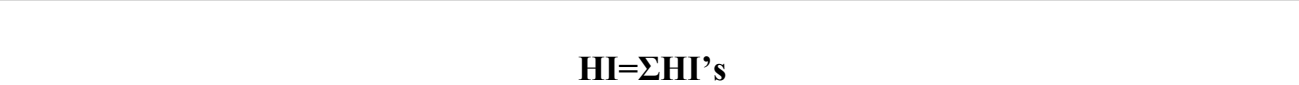 } & & $\begin{array}{c}25.6554 \\
1 \\
\end{array}$ \\
\hline
\end{tabular}




\begin{tabular}{|c|c|c|c|c|c|c|c|}
\hline 2 & Pyrethroids & Bifenthrin & 10 & 10 & JMPR_2009 & 0.003 & 0.02600 \\
\hline & & Cypermethrin & 20 & 40 & JMPR_2006 & 0.259 & 1.29251 \\
\hline & & Fenpropathrin & 30 & 30 & JMPR_2012 & 0.085 & 0.28320 \\
\hline & & $\begin{array}{l}\text { Lambda- } \\
\text { Cyhalothrin }\end{array}$ & 20 & 20 & JMPR_2007 & 0.113 & 0.56526 \\
\hline & & Permethrin & 50 & 50 & JMPR_1999 & 0.003 & 0.00624 \\
\hline \multicolumn{6}{|c|}{$H I=\Sigma H I ' s$} & & 2.17320 \\
\hline \multirow[t]{5}{*}{3} & Organochlorine & Atrazine & 20 & 100 & JMPR_ 2007 & 0.020 & 0.09983 \\
\hline & & Fenarimol & 10 & 0 & JMPR_1995 & 0.000 & 0.00028 \\
\hline & & Indoxacarb & 10 & 100 & JMPR_2005 & 0.039 & 0.39475 \\
\hline & & Pyridaben & 10 & 50 & EFSA 10 & 0.003 & 0.02658 \\
\hline & & Spirodiclofen & 10 & 10 & JMPR_2009 & 0.008 & 0.08320 \\
\hline \multicolumn{6}{|c|}{ 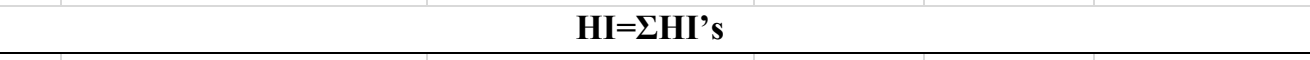 } & & 0.60465 \\
\hline \multirow[t]{7}{*}{4} & Carbamates & Carbendazim & 30 & 100 & JMPR_2005 & 0.158 & 0.52762 \\
\hline & & Carbofuran & 1 & 1 & JMPR_2008 & 0.019 & 1.85000 \\
\hline & & Carbosulfan & 10 & 20 & JMPR_2003 & 0.000 & 0.00000 \\
\hline & & Chlorpropham & 50 & 500 & JMPR_ 2005 & 0.009 & 0.01788 \\
\hline & & Propamocarb & 400 & 2000 & JMPR_2005 & 25.146 & 6.28654 \\
\hline & & Thiophanate-methyl & 80 & 80 & JMPR_2006 & 0.439 & 0.54823 \\
\hline & \multicolumn{5}{|c|}{ 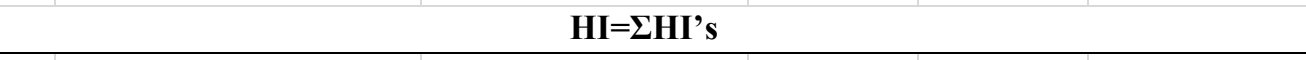 } & & 9.23028 \\
\hline \multirow[t]{23}{*}{5} & Heterocyclic & Acetamiprid & 70 & 100 & JMPR_2011 & 0.248 & 0.35402 \\
\hline & & Bromuconazole & 10 & 100 & 10/92/EU & 0.067 & 0.66977 \\
\hline & & Buprofezin & 8 & 500 & JMPR_ 2009 & 0.030 & 0.37254 \\
\hline & & Clothianidin & 100 & 600 & JMPR_2010 & 0.000 & 0.00000 \\
\hline & & Cyprodinil & 30 & 30 & JMPR_2003 & 0.001 & 0.00354 \\
\hline & & Difenoconazole & 10 & 300 & JMPR_2007 & 0.189 & 1.88941 \\
\hline & & Famoxadone & 6 & 600 & JMPR_2003 & 0.260 & 4.32614 \\
\hline & & Fenpyroximate & 10 & 20 & JMPR_2007 & 0.000 & 0.00245 \\
\hline & & Flusilazole & 7 & 20 & JMPR_2007 & 0.000 & 0.00457 \\
\hline & & Hexaconazole & 5 & 0 & JMPR_1990 & 0.000 & 0.00113 \\
\hline & & Hexythiazox & 30 & 30 & JMPR-2008 & 0.078 & 0.25941 \\
\hline & & Imidacloprid & 60 & 400 & JMPR_2001 & 0.444 & 0.74011 \\
\hline & & Iprodione & 60 & 0 & JMPR_1995 & 0.210 & 0.35038 \\
\hline & & Myclobutanil & 30 & 300 & JMPR_2014 & 0.019 & 0.06413 \\
\hline & & Penconazole & 30 & 0 & JMPR_1992 & 0.002 & 0.00526 \\
\hline & & Propiconazol & 70 & 300 & JMPR_2004 & 0.010 & 0.01419 \\
\hline & & Pyrimethanil & 200 & 0 & JMPR_2007 & 0.093 & 0.04673 \\
\hline & & Tebuconazole & 30 & 300 & MPR_2010 & 0.071 & 0.23506 \\
\hline & & Tetraconazole & 4 & 50 & EFSA 08 & 0.006 & 0.14550 \\
\hline & & Thiacloprid & 10 & 30 & JMPR_2006 & 0.095 & 0.95281 \\
\hline & & Thiamethoxam & 80 & 1000 & JMPR_2010 & 0.176 & 0.22052 \\
\hline & & Triadimenol & 30 & 80 & JMPR_2004 & 0.030 & 0.10090 \\
\hline & \multicolumn{5}{|c|}{$H I=\Sigma H I ' s$} & & 10.7585 \\
\hline
\end{tabular}




\begin{tabular}{|c|c|c|c|c|c|c|c|}
\hline \multirow{2}{*}{6} & & & & & & & 7 \\
\hline & \multicolumn{7}{|l|}{ Other } \\
\hline & Antihelmintics & $\begin{array}{l}\text { Emamectin } \\
\text { Benzoate }\end{array}$ & 1 & 20 & JMPR_2014 & 0.057 & $\begin{array}{c}11.4925 \\
0\end{array}$ \\
\hline & Benzoylurea & Lufenuron & 20 & 20 & JMPR_2015 & 0.014 & 0.07230 \\
\hline & Bezamide & Flutolanil & 90 & 90 & JMPR_2002 & 0.040 & 0.04462 \\
\hline & Dimethylaniline & Metalaxyl & 80 & 500 & 2010/28/EU & 0.363 & 0.45383 \\
\hline & Dinitrophenol & Pendimethalin & 100 & 1000 & JMPR_2016 & 0.014 & 0.01359 \\
\hline & Ethylurea & Cymoxanil & 13 & 80 & EFSA 08 & 0.033 & 0.25760 \\
\hline & Hydroxymethylamine & Spinosad & 20 & 20 & JMPR_2004 & 0.109 & 0.54450 \\
\hline & Methoxyacrylateate & Azoxystrobin & 200 & 200 & JMPR_2008 & 1.175 & 0.58743 \\
\hline & Morpholine & Dimethomorph & 200 & 600 & JMPR_2007 & 0.259 & 0.12955 \\
\hline & Methoxylimine & Trifloxystrobin & 40 & 40 & JMPR_2004 & 0.354 & 0.88424 \\
\hline & Phenylacetamide & Benalaxyl & 70 & 100 & JMPR_ 2005 & 0.012 & 0.01663 \\
\hline & Ynoxyacetamide & Mandipropamid & 200 & 200 & JMPR_2008 & 0.017 & 0.00873 \\
\hline & Trifluoromethylbenzene & Oxyfluorfen & 3 & 300 & $\begin{array}{l}(\mathrm{EU}) \\
2017 / 359\end{array}$ & 0.000 & 0.00094 \\
\hline & Yloxypyridine & Pyriproxyfen & 100 & 100 & JMPR_2001 & 0.002 & 0.00208 \\
\hline & & $H I=\Sigma H$ & & & & & $\begin{array}{c}14.5085 \\
4\end{array}$ \\
\hline
\end{tabular}

\section{CONCLUSION}

The monitoring survey studies of pesticide residues in Egyptian vegetable markets still poses special concern since of their use for some types of pesticides such as OP's and carbamates for long years. However, possible accumulation of these hazards in plant crops and environment components could cause considerable health problems for both famers and the consumers. Accordingly, the Egyptian authorization should strengthen their efforts on establish official control plan as well as a regular monitoring studies, to ensure following of local GAP and promote education on the potential risks and the safe use of pesticides. Moreover, cumulative risk assessment is considered as an indicator for the lethal effects that related to the human health because of the exposure to pesticide residues over a long period to multiple pesticide groups i.e. organophosphorus, organochlrine, pyrethroids and carbamates.

\section{Compliance with Ethical Standards}

Conflicts of interest: None

Ethics approval: This article does not involve any human or animal participants by any of the given authors

\section{REFERENCES}

[1] DIET, nutrition and the prevention of chronic diseases "report of a Joint WHO/FAO Expert Consultation, WHO Technical Report Series" No. 916. Geneva: World Health Organization, 2003. https://www.who.int/dietphysicalactivity/publications/trs916/en/ 
[2] Shakhaoat, H.,Alamgir, H., Abdur, R., Mainul, I., Atiqur, R., Tanveer, M.A. "Health risk assessment of pesticide residues via dietary intake of market vegetables from Dhaka, Bangladesh" Foods,Vol. 2, Issue 1, pp. 64-75, 2013. https://doi.org/10.3390/foods2010064

[3] FAO/WHO, Retrieved January 2013. http://www.codexalimentarius.net

[4] Khaniki, G.R. "Chemical contaminants in milk and public health concerns" A review. International Journalof Dairy Science, Vol. 2, Issue 2, pp. 104-115, 2007. https://scialert.net/abstract/?doi=ijds.2007.104.115

[5] Bhanti, M., Taneja, A. "Contamination of vegetables of different seasons with organophosphorus pesticides and related health risk assessment in northern India" Chemosphere, Vol. 69, Issue 1, pp. 63-68, 2007. https://doi.org/10.1016/j.chemosphere.2007.04.071

[6] Berrada, H., Fernandez, M., Ruiz, M. J., Molto, J. C., Manes, J., Font, G. "Surveillance of pesticide residues in fruits from Valencia during twenty months (2004/2005)" Food Control, Vol. 21, Issue 1, pp. 36-44, 2010. https://doi.org/10.1016/j.foodcont.2009.03.011

[7] Bhanti, M., ShuklaG., Taneja A., "Contamination levels of organochlorine pesticides andfarmers' knowledge, perception, practices in rural India-A case study"Bulletin of Environmental Contamination and Toxicology, Vol. 73, Issue 5, pp. 787-793, 2004. https://doi.org/10.1007/s00128-004-0496-8

[8] Ntow, W.J.,Gijzen,H.J., Kelderman,P.,Drechsel,P., "Farmer perceptions and pesticide use practices in vegetable production in Ghana" Pest Management Science, Vol. 62, Issue 4, pp. 356365, 2006. https://doi.org/10.1002/ps.1178

[9] Duck, W. P., Kwang, G. K., Eun, A. C., Gyeong, R. K., Tae, S. K.,Yong, S. Y., Bae, S. C. "Pesticide residues in leafy vegetables, stalk and stem vegetables from South Korea: A long-term study on safety and health risk assessment" Food Additives and Contaminants Part A, Vol. 33, Issue 1, pp. 105-118, 2016. https://doi.org/10.1080/19440049.2015.1108524

[10] Sohair, A. G. A., Almaz, M. M., Thabet, W. M., Nabil, M. M. "Evaluation of pesticide residues in some Egyptian fruits" International Journal of Environment, Vol. 4, Issue 1, pp. 87-97, 2015. http://www.curresweb.com/ije/ije/2015/87-97.pdf

[11] Hjorth, K. B. Johansen, A. Holen, Andersson,H.B., Christensen,K., Siivinen,M., Toome, "Pesticide residues in fruits and vegetables from South America e A Nordic project" Food Control, Vol. 22, Issue 11, pp. 1701-1706, 2011.https://doi.org/10.1016/j.foodcont.2010.05.017

[12] FAO 2002a "Submission and evaluation of pesticide residues data for the estimation of maximum residue levels in food and feed, 1st ed. Rome" Food and Agriculture Organization of the United Nations, FAO Plant Production and Protection Paper, No. 170, 2002. http://www.fao.org/ag/agp/agpp/pesticid/JMPR/ Download/FAOM2002.pdf

[13] Claeys, W.L., Jean-Francois,S., Bragard, C., Maghuin- Rogister, G., Luc,P. "Schiffers,B., Exposure of several Belgian consumer groups to pesticide residues through fresh fruit and vegetable consumption: Food Contaminants, Vol. 22, Issue 3, pp. 508-516, 2011. http://hdl.handle.net/2268/175292

[14] FAO/WHO "Codex Alimentarius Commission. Committee on Pesticide Residues, Guidelines for predicting dietary intake of pesticide residues, Global Environment Monitoring System-Food Contamination Monitoring and Assessment Programme (GEMS/Food)" WHO, 1997. https://apps.who.int/iris/handle/10665/63787

[15] Codex Alimentarius International Food standards. Codex Maximum Residue Limits (MRLs). http://www.fao.org/fao-who-codexalimentarius/codextexts/dbs/pestres/pesticides/en/

[16] European Commission. EU Pesticides database. Maximum Residue Limits (MRLs). https://ec.europa.eu/food/plant/pesticides/eu-pesticides $\underline{\text { database/public/?event=homepage } \& \text { language }=\mathrm{EN}}$ 
[17] Commission Codex Alimentarius. "Portion of commodities to which maximum residue limits apply and which is analyzed CAC/GL 41-1993" Joint FAO/WHO food standards program, Vol. 2, p. 391, 1993.file:///C:/Users/TALA/Downloads/CXA 004 1993e.pdf

[18] European Union, Foods of Plant Origin "Determination of pesticide residues using GC-MS and/or LC-MS/MS following acetonitrile extraction/partitioning and clean-up by dispersive SPE QuEChERS method" BS EN, 15662, 2008.

http://www.chromnet.net/Taiwan/QuEChERS_Dispersive_SPE/QuEChERS_\%E6\%AD\%90\%E7 \%9B\%9F\%E6\%96\%B9\%E6\%B3\%95_EN156622008_E.pdf

[19] Banerjee, K., Dasharath, P., Dasgupta, O. S., Patil, S. B., Patil S. H., Savant, R.,Adsule, P. G."Validation and uncertainty analysis of a multi-residue method for pesticides in grapes using ethyl acetate extraction and liquid chromatography-tandem mass spectrometry" Journal of Chromatography A, Vol. 1173, Issue 1-2, pp. 98-109, 2007.

https://doi.org/10.1016/j.chroma.2007.10.013

[20] Eurachem guide "The fitness for purpose of analytical methods - A laboratory guide to method validation and related topics" Eurachem 2nd ed., ISBN 978-91-87461-59-0, 2014. https://www.eurachem.org/images/stories/Guides/pdf/MV guide 2nd ed EN.pdf

[21] Eurachem guide "Quantifying uncertainty in analytical measurement" Eurachem 3d edition, ISBN978-0-948926-30-3, 2012. File:///C:/Users/amohammed/Documents/QUAM2012_P1.pdf

[22] Darko, G., Akoto, O. "Dietary intake of organophosphorous pesticide residues through vegetables from Kumasi, Ghana" Food Chemical Toxicology, Vol. 46, Issue 12, pp. 3703-3706, 2008.https://doi.org/10.1016/j.fct.2008.09.049

[23] WHO/GEMS/Food Cluster diets, 2012. https://extranet.who.int/sree/Reports?op=vs\&path=/WHO_HQ_Reports/G7/PROD/EXT/GEMS_c luster_diets_2012\&userid=G7_ro\&password=inetsoft123

[24] FAO/WHO "Pesticide residues in food and feed. Acceptable Daily Intake" Codex Alimentarius Commission, FAO/WHO Food standards, 2010.

http://www.fao.org/fao-who-codexalimentarius/codex-texts/dbs/pestres/en/

[25] EFSA, Scientific Colloquium Summary Report "Cumulative Risk Assessment of Pesticides to Human Health” 2013.http://www.efsa.europa.eu/en/supporting/doc/colloquiapesticides.pdf

[26] Hamilton, D. \& Crossley, S. "Pesticide Residues in Food and Drinking Water: Human Exposure and Risks" John Wiley and Sons Ltd. Chichester, England, ISBN: 978-0-470-09160-9, pp.378, 2004.https://www.wiley.com/enus/Pesticide+Residues+in+Food+and+Drinking+Water\%3A+Hu man+Exposure+and+Risks-p-9780470091609

[27] Oliver, G. R., Bolles, H. G., Shurdut, B. A. "Chlorpyrifos: Probabilistic assessment of exposure and risk" Neurotoxicology, Vol. 21, Issue 1-2, pp. 203-208, 2000.

https://pubmed.ncbi.nlm.nih.gov/10794401

[28] Qozowicka, B., Jankowska, M., \&Kaczy, N. P. "Pesticide residues in Brassica vegetables and exposure assessment of consumers" Food Control, Vol. 25, Issue2, pp. 561-575, 2012.

http://dx.doi.org/10.1016/j.foodcont.2011.11.017

[29] Rosa, M. G., Raquel, R. O., Beatriz, C. G. J., Simal, G. N. "Occurrence of fungicide and insecticide residues in trade samples of leafy vegetables" Food Chemistry, Vol.107, Issue 3, pp. 1342-1347, 2008.https://doi.org/10.1016/j.foodchem.2007.09.045

[30] Selim, M. T., El-Saeid, M. H., Al-Dossari, L. M. "Multiresidues analysis of pesticides using gas chromatography mass spectrometry: I-leafy vegetables. Research Journal of Environmental Sciences, Vol. 5, Issue 3, pp. 248-258, 2011. DOI: 10.3923/rjes.2011.248.258

[31] Dogheim, S. M., El-Marsafy, A. M., Gad Alla, S. A., Khorshid, M.A., Fahmy, S. M. "Pesticides and heavy metals levels in Egyptian leafy vegetables and some aromaticmedicinal plants" Food Additives and Contaminants, Vol. 21, Issue 4, pp. 323-330, 2004. 
http://dx.doi.org/10.1080/02652030310001656361

[32] Sohair, A. G. A., Wasfi, M. T., Emil, Y. S. "Monitoring and risk assessment of pesticide residues in some Egyptian vegetables"Middle East Journal of Applied Sciences, Vol. 3, Issue 4, pp. 216230, 2013. http://www.curresweb.com/mejas/mejas/2013/216-230.pdf

[33] Ashutosh, K. S., Purushottam, T., Srivastava, M. K., Lohani, M., Laxman, P. S. “ Monitoring of pesticide residues in market basket samples of vegetable from Lucknow City, India: QuEChERS method" Environmental Monitoring and Assessment, Vol. 176, Issue 1-4, pp. 465-472, 2011. DOI: $10.1007 / \mathrm{s} 10661-010-1597-\mathrm{y}$

[34] Hyum, H. N., Young, S. P., Kyung, W. K. P., Kwang, H. L., Jae, Y.L., Kyung, W. Y. Kee, S. K. "Monitoring of pesticide residues in leafy vegetables collected from wholesale and traditional markets in Cheongju" The Korean Journal ofPesticide Science, Vol. 14, Issue 2, pp. 381-393, 2010.http://www.koreascience.or.kr/article/JAKO201015537949207.pdf

[35] Godfred, D., \& Osei, A. "Dietary intake of organophosphorus pesticide residues through vegetables from Kumasi, Ghana” Food and Chemical Toxicology, Vol. 469, Issue 12, pp.37033706, 2008.https://doi.org/10.1016/j.fct.2008.09.049

[36] EFSA "Annual report on pesticide residues according to article 32 of regulation (EC) No 396/2005” EFSA Journal, Vol. 8, Issue 6, pp. 1646, 2010. https://efsa.onlinelibrary.wiley.com/doi/pdf/10.2903/j.efsa.2010.1646

[37] The Federal Institute for Risk Assessment (BfR), 2013. https://www.bfr.bund.de/en/press_information/2013/10/risk_at_a_glance-186393.html

[38] Gupta P.K."Pesticide exposure-Indian scene" Toxicology, Vol. 198, Issue 1-3, pp. 83-90, 2004. https://doi.org/10.1016/j.tox.2004.01.021 